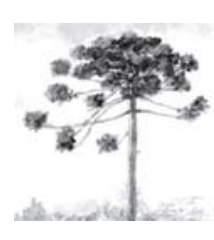

\title{
OCORRÊNCIA DE Cryptosporidium spp. EM PEIXES-BOI MARINHOS (Trichechus manatus) E FUNCIONÁRIOS ENVOLVIDOS NO MANEJO DA ESPÉCIE
}

\author{
Ocurrence of Cryptosporidium spp. in antillean manatee \\ (Trichechus manatus) and the staff involved in management of the species
}

\author{
João Carlos Gomes Borges ${ }^{1}$, Leucio Câmara Alves ${ }^{2}$, \\ Maria Aparecida da Gloria Faustino ${ }^{3}$, Ana Maria Alves Lima ${ }^{4}$
}

\footnotetext{
${ }^{1}$ Ph.D., Professor do Programa de Pós-Graduação em Ciência Veterinária. Universidade Federal Rural de Pernambuco, Aracaju, SE - Brasil, e-mail: jcgborges@hotmail.com

${ }^{2}$ Ph.D., Professor da Universidade Federal Rural de Pernambuco. Laboratório de Doenças Parasitárias dos Animais Domésticos, Aracaju, SE - Brasil, e-mail: leucioalves@hotmail.com

${ }^{3}$ Ph.D., Pofessora da Universidade Federal Rural de Pernambuco. Laboratório de Doenças Parasitárias dos Animais Domésticos, Aracaju, SE - Brasil, e-mail: magfaustino@hotmail.com

${ }^{4}$ Ph.D., Professora do Programa de Pós-Graduação em Ciência Veterinária. Universidade Federal Rural de Pernambuco, Aracaju, SE - Brasil, e-mail: alveslam@bol.com.br
}

\begin{abstract}
Resumo
A criptosporidiose é considerada mundialmente uma zoonose que pode afetar o homem e um grande número de animais domésticos e silvestres. Entretanto, a infecção em humanos ocorre por caráter antroponótico (entre humanos) ou zoonótico (animal para o homem ou vice-versa). Objetivou-se, no presente estudo, avaliar a relação entre a ocorrência de oocistos de Cryptosporidium spp. em peixes-boi marinhos (Trichechus manatus) e em funcionários envolvidos na manutenção destes animais em cativeiro. Para tanto, foram coletadas amostras de 28 peixes-boi marinhos e de 21 funcionários envolvidos nas diversas atividades relacionadas ao manejo destes animais, sendo estas amostras processadas a partir da sedimentação pelo formol-éter e o diagnóstico realizado pela técnica de Kinyoun. Após as análises, as amostras positivas foram submetidas ao Teste de Imunofluorescência Direta. Os resultados revelaram a ocorrência de oocistos de Cryptosporidium sp. em 23,21\% (26/112) das amostras de peixes-boi marinhos, sendo os filhotes os mais acometidos. No que concerne aos funcionários, os oocistos deste coccídio estiveram presentes em 23,80\% (10/42) do material analisado. A infecção por Cryptosporidium spp. em humanos pode constituir um caminho para a infecção de peixes-boi marinhos, durante os cuidados e manejo dos animais. De maneira semelhante, os animais infectados representam implicações para a saúde pública.
\end{abstract}

Palavras-chave: Sirênios; Transmissão; Coccídio; Humanos. 


\begin{abstract}
Cryptosporidiosis is world-wide a zoonosis which can affects man and a wide range of domestic and wild animals. However the infection in humans occurs by either anthroponotic (between humans) or zoonotico (animal to human or vice-versa). The objective in the present study was to verify the relationship between the occurrence of Cryptosporidium spp. in Antillean manatee (Trichechus manatus) and the staff involved directly in captive animal care and management. Samples from 28 Antillean manatees and 21 employees involved in the diverse activity with the management this animals were collected and processed by sedimentation by method employing formol-eter and the diagnosis were processed by Kinyon technique. At the end of the analyzing operations, the positive samples were submitted to the Direct Immunoflorescence Test. The results showed the occurrence of oocysts of Cryptosporidium spp. in 23,21\% (26/112) of the samples from the Antillean manatees, being the calves the most attempted by the parasite. About the employees, the oocysts of the coccidian were present in 23,80\% (10/42) of the analyzed samples. The infection of Cryptosporidium spp. in human may constitute a way to infection of Antillean manatees, during the animals care and management. In the same way, the infected animals may represent serious implications to public health.
\end{abstract}

Keywords: Sirenians; Transmission; Coccidian; Humans.

\section{INTRODUÇÃO}

Considerando as divergências existentes acerca da taxonomia do Cryptosporidium (1, 2, 3), atualmente 13 espécies são aceitas (4), sendo estas reportadas acometendo os répteis, peixes, aves e mamíferos, incluindo o homem $(5,6,7)$.

Entre as espécies deste protozoário, o Cryptosporidium parvum e o Cryptosporidium hominis apresentam a maior prevalência em humanos $(2$, 8), sendo estas espécies também descritas acometendo os mamíferos aquáticos $(9,10)$.

Nas populações de mamíferos aquáticos, os aspectos epidemiológicos relacionados à ocorrência deste agente ainda não estão bem esclarecidos (9), sobretudo o que diz respeito à origem de sua veiculação, o impacto da infecção na saúde destes animais e o papel destas espécies marinhas na transmissão do Cryptosporidium para o homem e outros animais (11).

Foi sugerido por Deng et al. (9) que algumas espécies atuam como reservatório do Cryptosporidium spp., a exemplo dos leõesmarinhos da Califórnia (Zalophus californianus). Além disso, a contaminação de águas marinhas por resíduos agropecuários, esgotos, descargas fecais de embarcações são alguns dos fatores apontados como meio de disseminação dos oocistos deste coccídio aos animais marinhos
(12), havendo, portanto, uma grande influência de distúrbios e interações antrópicas.

Suposições acerca da origem dos oocistos de Cryptosporidium nos animais marinhos foi corroborada com a identificação de Cryptosporidium hominis em dugongo (Dugong dugon), reforçando as evidências de que a infecção humana pode desencadear a infecção nestes animais (10), seja decorrente do contato entre estes por meio da rota fecal-oral ou da ingestão de água e alimentos contaminados.

Em alguns casos, após a infecção nos hospedeiros, a ação do coccídio pode desencadear alterações gastroentéricas (13), acarretando em diversas manifestações clínicas, conforme observado por Borges et al. (14), em peixe-boi marinho, porém no estudo não foram apresentados aspectos inerentes à origem da infecção e dos fatores epidemiológicos relacionados.

Tendo em vista que as estratégias de reabilitação e manutenção adotadas com os peixes-boi marinhos, como as atividades de manejo alimentar, limpeza de piscinas, biometrias, exames clínicos e translocações, requerem uma interação direta dos funcionários com estes animais, sendo algumas destas executadas por um número expressivo de pessoas, despertaram para a possibilidade do agente estar sendo veiculado a partir destas ocasiões. 
Desta forma, este trabalho teve por objetivo avaliar a relação entre a ocorrência de Cryptosporidium spp. em peixes-boi marinhos (Trichechus manatus) e os funcionários envolvidos na manutenção destes animais em cativeiro.

\section{MATERIAL E MÉTODOS}

\section{Coleta de material biológico}

\section{Animais}

As atividades foram realizadas no Centro Mamíferos Aquáticos/IBAMA-FMA, localizada na Ilha de Itamaracá ( $7^{\circ} 44^{\prime} 52^{\prime \prime}$ Sul; 34 49' 32" Oeste), estado de Pernambuco -Brasil, entre janeiro de 2005 a janeiro de 2007. Nesse local, eram mantidos 28 peixes-boi marinhos, sendo machos e fêmeas, com idade entre uma semana a 43 anos. Desses animais, nove estavam em piscinas de manutenção permanente, sendo que apenas um possuía idade inferior a um ano de idade. Os demais se encontravam em piscinas de reabilitação, onde em determinados momentos permaneciam individualmente ou em grupos de até cinco indivíduos, com a faixa etária compreendida entre filhotes e juvenis.

Para todos os animais eram ofertados capim-agulha (Halodule wrightii) e algas marinhas, sendo acrescentada cenoura e alface para animais mantidos nas piscinas de manutenção permanente, além de uma formulação láctea para os filhotes órfãos. Entretanto, de acordo com a idade desses animais, as estratégias de manejo adotadas eram distintas, a exemplo da quantidade de biometrias realizadas, avaliações clínicas e amamentação, onde a freqüência diminuía ao longo do tempo.

Todos os animais foram submetidos a exames clínicos prévios e na ocasião da biometria realizou-se a coleta de fezes. Este procedimento ocorreu em quatro ocasiões para cada espécime, em intervalos de 60 dias, totalizando 112 amostras fecais. O material foi acondicionado em frascos contendo solução constituída de álcool, formol, ácido acético glacial e água destilada (AFA), de acordo com o sugerido por Ueno e Gonçalves (15). Os frascos foram devidamente identificados e encaminhados ao Laboratório de Doenças Parasitárias dos Animais Domésticos (LDPAD), da Universidade Federal Rural de Pernambuco.

\section{Funcionários}

No intuito de esclarecer os aspectos inerentes a criptosporidiose, foi ministrada uma palestra aos funcionários do CMA/IBAMA-FMA, com novas informações transmitidas a estes durante o período das atividades, de acordo com o interesse e as dúvidas apresentadas. Posteriormente, contando com a participação espontânea de 21 destes, foram encaminhadas ao LDPAD 42 amostras fecais inerentes a duas coletas de cada pessoa, realizadas em intervalos de 21 dias.

$\mathrm{Na}$ ocasião em que as amostras foram entregues, de maneira informal, foi perguntado aos funcionários sobre alguma manifestação clínica compatível à ação do coccídio, além disso, foi constatado o enquadramento profissional deles, que corresponderam a tratadores de peixesboi, mergulhadores para coleta de capim-agulha, monitores da qualidade de água das piscinas, serviços gerais, estagiários e médicos veterinários. De acordo com as atribuições requeridas, a interação destes com os animais apresentavam intensidades distintas, porém todos apresentavam algum tipo de contato.

\section{Processamento laboratorial}

\section{Animais}

Inicialmente, as amostras fecais provenientes dos peixes-boi marinhos foram submetidas à sedimentação pelo formol-éter com posterior confecção dos esfregaços e coloração por meio da técnica de Kinyoun (16), sendo as amostras positivas submetidas, posteriormente, a imunofluorescência direta conforme recomendação do fabricante do kit Merifluor ${ }^{1}$.

Para a identificação dos oocistos, nas preparações com coloração de Kinyoun sob leitura com microscopia óptica, consideraram-se as estruturas ovóides ou esféricas de tamanho compatível a 4-6 $\mu \mathrm{m}$ de diâmetro, que

${ }^{1}$ Meridian Bioscience Diagnostics, Cincinnatti, Ohio 
apresentavam coloração vermelha e estruturas internas (17), enquanto que na análise de imunofluorescência direta, consideraram-se as estruturas com tamanho e formato compatíveis, na cor verde-maçã, com ou sem estruturas exibindo fluorescência, conforme Sterling e Arrowood (18).

\section{Funcionários}

Posteriormente às análises do material proveniente dos peixes-boi marinhos, procedeuse o processamento das amostras fecais dos funcionários, sendo utilizado o mesmo método descrito anteriormente para a pesquisa de Cryptosporidium spp.

Além das técnicas utilizadas para o diagnóstico da criptosporidiose, as amostras foram submetidas ao processamento pelo método direto (19), flutuação $(19,20)$ e sedimentação $(21,22)$, no intuito de ser avaliada a presença de outros agentes parasitários.

\section{ANÁLISE ESTATÍSTICA}

Para avaliar a possibilidade de existir correlação entre a idade dos animais e a ocorrência da infecção por Cryptosporidium spp. nos peixes- boi marinhos, foi utilizado o teste de Spearman (23), a partir dos dados ordenados. De modo semelhante, para avaliar a correlação entre o grau de exposição dos funcionários aos peixes-boi marinhos e a ocorrência da infecção por Cryptosporidium spp., foi utilizado o mesmo teste de Spearman $(\mathrm{p}<0,05)$.

\section{RESULTADOS E DISCUSSÃO}

Com base no material fecal proveniente dos peixes-boi marinhos, foram observados pelo método de Kinyoun oocistos de Cryptosporidium spp. em 23,21\% (26/112) das amostras analisadas, sendo estas confirmadas pelo teste de imunofluorescência direta. Estes resultados foram referentes a 46,42\% (13/28) dos peixes-boi marinhos avaliados e, de acordo com a faixa etária dos animais estudados, os filhotes mostraram-se mais susceptíveis à infecção pelo agente (Tabela 1). Entre os animais com diagnóstico positivo, foi observado que $76,92 \%$ $(10 / 13)$ apresentaram, ao longo do período de coleta das amostras fecais, algum sinal clínico sugestivo à ação do agente, tais como quadros de diarréia, desconforto abdominal, aumento do intervalo respiratório, letargia e emagrecimento.

TABELA 1 - Freqüência absoluta e ralativa de peixes-boi marinhos infectados por Cryptosporidium spp. nas diferentes faixas etárias

\begin{tabular}{lcccc}
\hline Faixa Etária & Número deAnimais & FA & FR $(\%)$ & Animais comCryptosporidum $(\%)$ \\
\hline Adulto & 8 & 3 & 23,07 & $37,50(03 / 08)$ \\
Juvenis & 6 & 2 & 15,39 & $33,33(02 / 06)$ \\
Filhotes & 14 & 8 & 61,54 & $57,14(08 / 14)$ \\
Total & 28 & 13 & 100 & - \\
\hline
\end{tabular}

FA (Freqüência Absoluta); FR (Freqüência Relativa)

A freqüência encontrada da infecção por Cryptosporidium spp. em peixes-boi marinhos cativos representa um valor expressivo, sobretudo pela facilidade em ser disseminada aos demais animais, constituindo assim como um risco eminente à saúde dos demais espécimes cativos.

Em decorrência da ausência de estudos semelhantes desenvolvidos anteriormente com esta espécie, de maneira a possibilitar avaliações comparativas, foram utilizadas como base as pesquisas realizadas com outros mamíferos aquáticos. Dessa forma, o resultado inerente à infecção por Cryptosporidium constatado neste estudo apresentou-se ligeiramente acima da média encontrada com outras espécies, sendo relatada a prevalência de $11 \%$ em elefante-marinho do norte (Mirounga angustirostris) e foca-comum (Phoca vitulina) (12), 50\% em leões-marinhos da Califórnia (9), 5,1\% em baleia da Groenlândia (Balaena mysticetus), 22,6\% em foca-anelada (Phoca bispida) e $24,5 \%$ em baleia-franca do norte, Eubalaena glacialis (12). 
A freqüência da infecção por Cryptosporidium spp. entre os filhotes de peixesboi marinhos difere das constatações observadas por Hughes-Hanks et al. (12), onde relataram que em populações de foca-anelada, a faixa etária mais acometida foi de indivíduos com idade superior a sete anos. Entretanto, em diversas espécies de mamíferos terrestres, a prevalência da criptosporidiose tem sido maior em filhotes, assim como a apresentação de sinais clínicos mais severos $(24,5,25,26)$.

Em analogia à ocorrência de Cryptosporidium spp. em bezerros, a maior susceptibilidade destes foi atribuída à presença de infecções concomitantes, à imaturidade do sistema imune e ainda aos fatores relacionados ao manejo, como a interação direta entre os animais, a alta densidade nas instalações, a qualidade da água e os alimentos ofertados (27, 28). Colaborando com estas informações, foi sugerido por Borges et al. (29) que a infecção de peixe-boi marinho pode ser resultante $\mathrm{da}$ contaminação dos ambientes costeiros, da oferta de itens alimentares, da manipulação de alimentos por tratadores e do contato direto destes com os animais.

Dessa forma, estas constatações justificam a infecção em filhotes de peixes-boi marinhos provenientes do hábitat e atualmente mantidos em processo de reabilitação, quando imediatamente após as atividades de resgate de quatro espécimes foi possível coletar e analisar amostras fecais antes destes serem encaminhados às piscinas de reabilitação, sendo realizadas posteriormente três coletas, com intervalos de 60 dias. Inicialmente todas as amostras apresentaram os primeiros resultados negativos, posteriormente dois animais apresentaram a infecção a partir do terceiro exame e nos dois outros indivíduos, somente foi diagnosticado o agente na quarta análise. Sendo assim, a infecção destes animais foi relacionada a fatores ligados ao manejo.

Com isso, além da possibilidade de veiculação dos oocistos para estes animais por meio da água e alimentos contaminados, acreditase que o contato destes com humanos, sobretudo os funcionários envolvidos no manejo, pode ter constituído como meio importante de veiculação do agente, em decorrência das práticas de higiene inadequada, haja vista que os filhotes permaneciam em piscinas individuais nas etapas iniciais de reabilitação.

Para avaliar a influência das atividades de manejo nos peixes-boi marinhos, foram agrupados os espécimes em processo de reabilitação (compreendendo as categorias de idade de filhotes e juvenis), em virtude da semelhança da rotina adotada, no que diz respeito à alimentação, características das piscinas, contato com tratadores e técnicos. Nesta análise, foi utilizada a freqüência de biometrias, como indicador da intensidade de manejo (Tabela 2).

TABELA 2 - Freqüência de biometrias e infecção por Cryptosporidium spp. em peixes-boi marinhos em reabilitação

\begin{tabular}{lccc}
\hline Idade dosAnimais & Freqüência das biometrias & Número total de biometrias no período & Infecção por Cryptosporidium spp. \\
\hline 1 mês & Semanal & 4 & $0 \%(00 / 08)$ \\
$2-3$ meses & Quinzenal & 42 & $5 \%(02 / 08)$ \\
$4-12$ meses & Mensal & 9 & $50 \%(04 / 08)$ \\
$13-36$ meses & Bimestral & 12 & $70 \%(07 / 10)$ \\
\hline
\end{tabular}

O teste estatístico de Spearman indicou a existência de correlação negativa entre a freqüência do manejo e o porcentual da infecção de Cryptosporidium spp. nos peixes-boi marinhos em processo de reabilitação $(\mathrm{r}=-0,9986 ; \mathrm{p}=<0,001)$. Nesse sentido, no transcorrer do processo de reabilitação, quanto maior a exposição dos animais ao manejo, de forma diretamente proporcional foi observada a infecção pelo Cryptosporidium spp.
No Brasil, entre as atividades conservacionistas em beneficio dos peixes-boi marinhos, vem sendo realizada de forma bem intencionada a reintrodução de espécimes após o processo de reabilitação (30). Porém, a partir da ocorrência de Cryptosporidium spp. em alguns animais cativos desperta para os riscos do coccídio ser disseminado para as populações nativas, conforme observado na transmissão de outros patógenos em diversas espécies animais (31). 
No que concerne aos funcionários, de acordo com a morfologia, coloração e tamanho, oocistos de Cryptosporidium spp. foram observados em 23,80\% (10/42) do material analisado, pelo método de Kinyoun e submetido à confirmação pelo teste de imunofluorescência direta.

Com base nas atividades desenvolvidas, os tratadores apresentaram o maior porcentual de pessoas com a infecção pelo coccídio, entre as categorias analisadas (Tabela 3).

TABELA 3 - Grau de Exposição e Freqüência da infecção nos diferentes enquadramentos profissionais de funcionários envolvidos nas atividades de manejo dos peixes-boi marinhos

\begin{tabular}{lccccc}
\hline Enquadramento Funcional & Grau de Exposição & Número de Funcionários & FA & FR (\%) & Funcionários com Cryptosporidium (\%) \\
\hline Tratadores & Alta & 4 & 4 & 40 & 100 \\
Mergulhadores & Baixa & 3 & 1 & 10 & 33,34 \\
Monitores da Qualidade & Alta & 4 & - & - & - \\
de $\mathrm{H}_{2} \mathrm{O}$ & & & 4 & 40 & 80 \\
Serviços Gerais & Baixa & 5 & - & - & - \\
Estagiários & Baixa & 3 & 1 & 10 & 50 \\
Veterinários & Média & 2 & 10 & 100 & - \\
Total & - & 21 & & & \\
\hline
\end{tabular}

Baixa (até 15h/mês); Média (16-80h/mês); Alta (acima de 80h/mês) FA (Freqüência Absoluta); FR (Freqüência Relativa)

Foi constatada, ainda, a presença de outros agentes parasitários, como Ancylostoma sp. e/ou Ascaris lumbricoides em 33,34\% (14/42) das amostras analisadas, onde a associação destes com os oocistos de Cryptosporidium spp. ocorreu em 7,14\% (03/42) das ocasiões. Entre as informações obtidas dos funcionários, em nenhuma ocasião foi mencionado algum tipo de manifestação clínica que pudesse ser relacionada à ação do agente.

Morgan et al. (10), após identificarem a ocorrência de Cryptosporidium hominis em dugongos, evidenciaram que outros mamíferos aquáticos também podem infectar-se e constituir como reservatório para a criptosporidiose humana, o que torna um fator de preocupação a presença deste coccídio em peixes-boi marinhos.

$\mathrm{O}$ resultado da análise de correlação utilizando o teste de Spearman indicou que não houve correlação entre o grau de exposição dos funcionários aos animais e a ocorrência de Cryptosporidium spp. ( $\mathrm{rs}=0,4058 ; \mathrm{t}=0,8881 ; \mathrm{p}=$ $0,4246)$. Porém, mesmo não sendo evidenciado pelas análises estatísticas existir correlação, em virtude das atribuições relacionadas ao manejo dos peixes-boi marinhos, que acarretam em alta exposição dos tratadores a estes animais, pode-se justificar a presença da infecção em todos os funcionários deste enquadramento profissional.
Relação semelhante foi descrita por Fayer e Ungar (32) e Levine et al. (33) ao atribuírem a infecção em estudantes de veterinária e tratadores ao provável contato com animais infectados.

No entanto é válido destacar que mesmo apresentando alto contato com os peixes-boi e ainda com os resíduos fecais, durante os procedimentos de limpeza das piscinas, não foi observada infecção nos monitores da qualidade de água. Em contrapartida, o alto porcentual da infecção nos funcionários de serviços gerais, mesmo com a baixa exposição, demonstra que outros fatores além do contato com os animais podem desencadear a infecção. Este fato ficou evidente após avaliar as amostras fecais provenientes de um funcionário desta última categoria mencionada, que recém-ingressava na Instituição, não havendo apresentado nenhum tipo de contato anterior com os peixes-boi, no entanto já apresentava a infecção pelo coccídio.

Ao término das atividades laboratoriais, foi recomendada a busca por serviços médicos especializados aos funcionários que apresentaram a infecção pelo Cryptosporidium spp., no entanto somente a adoção de protocolos terapêuticos prescritos não serão eficientes, haja vista a ausência de informação prévia sobre a criptosporidiose humana, bem como fatores relacionados na disseminação e controle por boa 
parte dos envolvidos. Além disso, a associação do Cryptosporidium spp. a outros agentes parasitários pode representar limitações nas medidas de higiene pessoal adotadas por estes.

Sendo assim, torna-se oportuna a realização de exames constantes em todos os funcionários que apresentem qualquer tipo de contato com os animais, bem como a elaboração de novas atividades de caráter informativo sobre a criptosporidiose, com abordagens específicas aos diferentes perfis dos profissionais envolvidos. Por meio destes conhecimentos adquiridos por parte de todos os funcionários envolvidos, haverá a possibilidade de consolidar a adoção de medidas que favoreçam o controle e a disseminação do Cryptosporidium, seja por meio da higiene pessoal ou nos cuidados relacionados aos animais.

\section{CONCLUSÕES}

A presença de humanos com a infecção por Cryptosporidium spp. pode constituir como meio de veiculação dos oocistos aos peixes-boi marinhos, por meio do contato realizado nas atividades de manejo. De maneira semelhante, os animais infectados representam implicações para a saúde pública, pois podem disseminar oocistos e vir a desencadear a infecção nos funcionários, constituindo, assim, como um novo reservatório para este agente.

\section{AGRADECIMENTOS}

Os autores agradecem a Jociery Einhardt Vergara-Parente, pela contribuição na coleta do material biológico, e a Cristiano Parente, pelas análises estatísticas. Ao Dr. Jean Carlos Ramos da Silva e Dra. Erilane Lima de Castro Machado, pelos comentários no manuscrito; a Régis Pinto de Lima e Denise Freitas de Castro, pelo apoio Institucional, respectivamente por meio do Centro Mamíferos Aquáticos/IBAMA e a Fundação Mamíferos Aquáticos; ao Programa de Pós-Graduação em Ciência Veterinária da UFRPE; à FACEPE e a Petrobras, por meio de patrocínio ao projeto Conservação de Matas Alagadas de Mamirauá.

\section{REFERÊNCIAS}

1. Morgan UM, Thompson RCA. PCR detection of Cryptosporidium, the way forward? Parasitology Today. 1998;14:241-245.

2. Sulaiman IM, Xiao L, Yang C, Escalante L, Moore A, Beard CB, et al. Differentiating human from animal isolates of cryptosporidium parvum. Emerging Infectious Diseases. 1998;4(4):681-685.

3. Xiao L, Sulaiman IM, Ryan UM, Zhou L, Atwill ER, Tischler ML, et al. Host adaptation and host-parasite co-evolution in Cryptosporidium: implications for taxonomy and public health. International Journal for Parasitology. 2002;32:1773-1785.

4. Xiao L, Fayer R, Ryan U, Upton SJ. Cryptosporidium taxonomy: recent advances and implications for public health. Clinical Microbiolology Reviews. 2004;17(1):72-97.

5. O’Donoghue PJ. Cryptosporidium and cryptosporidiosis in man and animals. International Journal Parasitology. 1995; 25:139-195.

6. Alvarez-Pellitero P, Sitjà-Bobadilla A. Cryptosporidium molnari n. sp. (Apicomplexa: Cryptosporidiidae) infecting two marine fish species, Sparus aurata L. and Dicentrarchus labrax L. International Journal for Parasitology. 2002;32:1007-1021.

7. Fayer R. Cryptosporidium: a water-borne zoonótico parasite. Veterinary for Parasitology. 2004;1-19.

8. Gonçalves EMN, Silva AJ, Eduardo MBP, Uemura IH, Moura INS, Castilho VLC, et al. Multilocus Genotyping of Cryptosporidium hominis associated with diarrhea outbreak in a dog care unit in São Paulo. Clinics. 2006;61(2):119-126.

9. Deng M, Peterson RP, Cliver DO. First findings of Cryptosporidium and Giardia in California sea lions (Zalophus californianus). Journal of Parasitology. 2000;86:490-494.

10. Morgan UM, Xiao L, Hill BD, O’Donoghue P, Limor ALAJ, Thompson ARC. Detection of the Cryptosporidium parvum "Human" Genotype in a Dugong (Dugong dugon). Journal of Parasitology. 2000;86:1352-1354. 
11. Santín M, Dixon BR, Fayer R. Genetic Characterization of Cryptosporidium Isolates From Ringed Seals (Phoca hispida) in Northern Québec, Canada. Journal of Parasitology. 2005;91(3):712-716.

12. Hughes-Hanks JM, Rickard LG, Panuska C, Saucier JR, O'Hara TM, Dehn L, Rolland M. Prevalence of Cryptosporidium spp. and Giardia spp. in Five Marine Species. Journal of Parasitology. 2005;91(5):1255-1228.

13. Hill BD, Fraser IR, Prior HC. Cryptosporidium infection in a dugong (Dugong dugon). Australia Veterinary Journal. 1997; 75(9):670-671.

14. Borges JCG, Lima EC, Alves LC, VergaraParente JE, Faustino MAG, Lima AMA, et al. Cryptosporidium spp em peixe-boi marinho (Trichechus manatus manatus) cativo no Centro Mamíferos Aquáticos, Ilha de Itamaracá, no estado de Pernambuco. In: $1^{\circ}$ Congresso Nacional de Saúde Pública Veterinária; 2005; Pernambuco, Brasil, Pernambuco; 2005. p. 35.

15. Ueno H, Gonçalves PC. Manual para Diagnóstico das Helmintoses de Ruminantes. Porto Alegre: UFRGS; 1994. 163 p.

16. Brasil. Ministério da Saúde. Infecções oportunistas por parasitas em AIDS: técnicas de diagnóstico. Brasília; 1996. 27p.

17. Lima EC, Melo VSP, Brito FLC, Alves LC, Stamford TLM. Avaliação de diferentes técnicas de coloração histoquímica na identificação de oocistos de Cryptosporidium spp. em amostras de água e leite. Revista Brasileira de Ciência Veterinária. 2004; 11(1/2):21-26.

18. Sterling CR, Arrowood MJ. Detection of Cryptosporidium sp. Infections using a direct immunofluorescente assay. The Pediatric Infectious Disease Journal. 1986;5:139-142.

19. Urquhart GM, Armour J, Duncan JL, Dunn AM, Jennings FW. Parasitologia Veterinária. Rio de Janeiro: Guanabara Koogan; 1996.
20. Willis HH. A simple levitation method for the detection of hookworm ova. Medical Journal Australia 1927;375-376.

21. Watanabe S, Nagayama I, Iwata K. A simple method for detection of Fasciola ova in bovine feces. Journal Veterinary Medical Association. 1953;2(6):176-177.

22. Sloss MW, Zajac AM, Kemp RL. Parasitologia Clínica Veterinária. São Paulo: Manole; 1999.

23. Callegari-Jacques SM. Bioestatística. Principios e Aplicações. Porto Alegre: Artmed; 2003. 255 p.

24. Dubey JP, Speer CA, Fayer R. Cryptosporidiosis of man and animals. Boston: CRC; 1990.

25. Pena HFJ, Kasai N, Gennari SM. Cryptosporidium muris in dairy cattle in Brazil. Veterinary for Parasitology. 1997;73:353-355.

26. Fuente R, Luzón JAH, Quiteria RS, Cid AGD, Orden JA, García S, et al. Cryptosporidium and concurrent infections with other major enterophatogens in 1 to 30-day-old diarrheic dairy calves in central Spain. Veterinary for Parasitology. 1999;80:179-185.

27. Maldonado-Camargo S, Atwill ER, SaltijeralOaxaca JA, Herrera-Alonso LC. Prevalence of and risk factors for shedding of Cryptosporidium parvum in Holstein Freisian dairy calves in central México. Preventive Veterinary Medicine. 1998;36:95-107.

28. Becher KA, Robertson ID, Fraser DM, Palmer DG, Thompson RCA. Molecular epidemiology of Giardia and Cryptosporidium infections in dairy calves originating from three souces in Western Australia. Veterinary for Parasitology. 2004;123:1-9.

29. Borges JCG, Alves LC, Marmontel M, Lima DS, Castro EL, Vergara-Parente JE, et al. Infecção por Cryptosporidium spp.: uma ameaça à conservação dos peixes-boi marinhos (Trichechus manatus manatus) e amazônicos (Trichechus inunguis). In: Resumos do $7^{\circ}$ Congresso Internacional sobre Manejo de Fauna Silvestre na Amazônia e América Latina, Ilhéus: UESC; 2006. p. 29. 
30. Lima RP, Alvite CMC. Protocolo de Reintrodução de Peixes-boi Marinhos (Trichechus manatus manatus) no Brasil. In: Resumos do $1^{\text {a }}$ Reunión Internacional sobre el Estudio de los Mamíferos Acuáticos SOMEMMA - SOLAMAC; México: SOLAMAC; 2006. p. 73.

31. Marini MA, Filho JSM. Translocação de aves e mamíferos: Teoria e prática no Brasil. In: Rocha CFD, Bergallo HG, Sluys MV, Alves MAS. Biologia da Conservação - Essencias. São Carlos: Rima; 2006. p. 505-536.
32. Fayer R, Ungar BLP. Cryptosporidium spp. and cryptosporidiosis. Microbiological Review. 1986;50:456-483.

33. Levine JF, Levy MG, Walker RL. Cryptosporidiosis in Veterinary student. Journal of the American Medicine Veterinary Association. 1988,193:1413-1414.

Recebido: 05/09/2006

Received: 09/05/2006

Aceito: 18/10/2006

Accepted: 10/18/2006 